\title{
1 Heritable gene expression variability governs clonal heterogeneity in
}

2 circadian period

3

$6 \quad{ }^{1}$ Laboratory of Chronobiology, Charité-Universitätsmedizin Berlin, Berlin, Germany.

$7{ }^{*}$ Corresponding author (achim.kramer@charite.de)

8

9 Short title: heritable basis of clonal circadian period heterogeneity

10 


\section{$11 \quad$ ABSTRACT}

12 A ubiquitous feature of circadian clocks across life forms is its organization as a network of

13 coupled cellular oscillators. Individual cellular oscillators of the network often exhibit a

14 considerable degree of heterogeneity in their intrinsic periods. While the interaction of coupling

15 and heterogeneity in circadian clock networks is hypothesized to influence clock's entrainability,

16 our knowledge of mechanisms governing network heterogeneity remains elusive. In this study,

17 we aimed to explore the principles that underlie inter-cellular period variation in circadian clock

18 networks (clonal period-heterogeneity). To this end, we employed a laboratory selection

19 approach and derived a panel of 25 clonal cell populations exhibiting circadian periods ranging

20 from $22 \mathrm{~h}$ to $28 \mathrm{~h}$. We report that while a single parent clone can produce progeny clones with a

21 wide distribution of circadian periods, heterogeneity is not entirely stochastically driven but has

22 a strong heritable component. By quantifying the expression of 20 circadian clock and clock-

23 associated genes across our panel, we found that inheritance of different expression patterns in

24 at least three clock genes might govern clonal period-heterogeneity in circadian clock networks.

25 Furthermore, we provide preliminary evidence suggesting that epigenetic variation might 26 underlie such gene expression variation.

\section{INTRODUCTION}

29 The majority of life forms on earth exhibit $\sim 24 \mathrm{~h}$ (circadian) behavioural and physiological rhythms generated by endogenous time-keeping mechanisms - circadian clocks. In addition to

31 driving such endogenous rhythms, circadian clocks facilitate synchronization of organisms'

32 rhythms to daily and seasonal changes in the environment to enhance their survivability, thereby 
functioning as an adaptive mechanism (Kumar, 2017). The fundamental basis of circadian

rhythm-generation across all life-forms are cell-autonomous molecular oscillations comprising (Dunlap, 1999). In higher organisms, such cell-autonomous clocks often function as a network of coupled oscillators which in unison drive circadian rhythms (Bell-Pedersen et al., 2005). Welsh and co-workers first reported that neurons within the suprachiasmatic nucleus (SCN; the master pacemaker in the hypothalamus of mammals) are surprisingly heterogeneous in their intrinsic periods of circadian firing pattern (Welsh et al., 1995). Subsequent studies revealed that such period-heterogeneity is not restricted to the SCN alone, but is also characteristic of mammalian peripheral clock cells (Nagoshi et al., 2004; Leise et al., 2012) as well as of clock cells in Drosophila (Sabado et al., 2017) and plants (Yakir et al., 2011; Muranaka and Oyama, 2016). The ubiquity of this network feature suggests that heterogeneity may be functionally relevant for circadian clocks (Jagota et al., 2000; Schaap et al., 2003; Gonze et al., 2005; Bernard et al., 2007; Inagaki et al., 2007; VanderLeest et al., 2007; Gu et al., 2016, 2019), thus likely being a substrate for natural selection. Interestingly, the observed period-heterogeneity among circadian clock cells within an organism cannot be entirely attributed to functionally different cell types as cells of the same subtype (clonal cells) also exhibit such variation (Nagoshi et al., 2004; Leise et al., 2016). Clonalheterogeneity or clonal-phenotypic variability is common in biology and can stem from various external factors such as stochastic changes in the microenvironment or internal factors like

52 stochastic partitioning of cellular components during cell-division or stochasticity in gene expression (Neildez-Nguyen et al., 2008; Brock, Chang and Huang, 2009; Huang, 2009; Altschuler 
55 Evans et al., 2018). In this study, we aimed to explore the possible mechanisms underlying clonal-

56 heterogeneity of circadian period in human circadian oscillator cells.

57 We hypothesised that clonal period-heterogeneity in mammalian cells is due to a) stochastic

58 variation (Geva-Zatorsky et al., 2006; Chang et al., 2008; Brock, Chang and Huang, 2009; Frank

59 and Rosner, 2012) and/or b) heritable variation (Dubnau and Losick, 2006; Gordon et al., 2009,

60 2013). Since the term 'stochastic' is used in the context of both non-heritable (external noise and

61 gene expression noise) as well as heritable gene expression variation (epigenetic stochasticity),

62 for the rest of this manuscript we define 'stochasticity' as any non-heritable variation (both

63 internal and external). To test the two hypothesis outlined above, we employed a laboratory

64 selection approach and derived a panel of 25 clonal cell lines (from a common ancestral/founding

65 culture) exhibiting a range of periods between $22 \mathrm{~h}$ and $28 \mathrm{~h}$. We observed that the period-

66 heterogeneity among progeny clones stemming from a single parent cell is not entirely stochastic

67 but has a substantial heritable component. We then measured expression of 20 clock and clock-

68 associated genes in our panel and observed that variation in gene expression levels of at least

69 three clock genes (transcription factors) might underlie clonal period-heterogeneity.

70 Furthermore, we provide preliminary evidence that epigenetic variation might govern the

71 observed clonal-variation in gene expression. 
76

77

78

79

80

81

82

83

84

85

86

87

88

89

90

91

92

\section{RESULTS}

\section{Clonal period-heterogeneity is not entirely stochastically driven but is largely inherited}

Is the variation in period among individual circadian oscillator cells just due to intrinsic and/or extrinsic stochastic noise? Or is there a heritable component? To test this, we single-cell cloned a 'founding culture' of U-2 OS cells (an established model of peripheral circadian clocks) harboring a BMAL1-luciferase reporter construct (Maier et al., 2009). Upon reaching confluence, the period of bioluminescence rhythms from these progeny cultures was determined by live-cell bioluminescence recording. As expected, we observed a distribution of circadian periods ( $23.5 \mathrm{~h}$ - $27.5 \mathrm{~h}$; Figure 1a top panel). We repeated this protocol for several 'assay-generations' by each time selecting short and long period cultures as 'parents' for the next assay-generation (Study outline in Supplementary Figure S1).

Interestingly, by repeating this protocol for several assay-generations we observed a directional divergence of the progeny period-distributions on either side of the 'founding culture's' distribution (Figure 1a). The mean circadian periods of progeny cultures in every assaygeneration were always very similar to those of their parental cultures (Figure 1a). Over the course of the selection protocol, the periods of short and long period clonal lines (SCL and $L C L$ ) significantly diverged from each other and from the 'founding culture'. The periods of both SCLs and LCLs diverged significantly by assay generation-2 and this divergence reached saturation as the periods did not diverge further ( $p<0.001$; ANOVA followed by Unequal N HSD; Figure 1b). At assay generation 4 , the circadian periods of LCLs were $\sim 3.4 \mathrm{~h}$ longer, and those of SCLs were $\sim 1.7$ shorter than the 'founding culture's' period (Figure 1b; Supplementary Figure S2). 
97 As a measure of period-heritability, we regressed mean periods of the progeny cultures on parental cultures and observed that parental period is a very good predictor of the mean progeny period $\left(R^{2}=0.96\right.$; Figure $\left.1 c\right)$. In addition, even though the divergence of period saturated over the last three assay generations (Figure 1b), we still observe a distribution of periods even after three assay generations. We reason that this distribution might be due to non-heritable stochasticity. Taken together, our results suggest that clonal period-heterogeneity might partly be stochastically driven but has a significant heritable component.

\section{Inheritance of differential gene expression levels might underlie period heritability}

We further aimed to explore the likely basis for heritability of the circadian period underlying

114 bioluminescence intensity can, in-principle serve as a proxy for the average expression level of the underlying gene (BMAL1 in this case) and hypothesized that clonal inheritance of average 
118

119

120

121

122

123

124

125

126

127

128

129

130

131

132

133

134

135

136

137

progeny values $\left(R^{2}=0.76\right.$; Figure $\left.2 a\right)$, while relative amplitude $\left(R^{2}=0.04\right.$; Supplementary Figure S3b) and damping rate $\left(R^{2}=0.40\right.$; Supplementary Figure $\left.S 3 c\right)$ were only poor predictors.

\section{Differential expression of E-Box associated factors may govern clonal period-heterogeneity}

To test, whether gene expression correlates with clonal period-heterogeneity, we used the NanoString multiplex technology to measure the average expression levels of 20 clock and clockassociated genes (Supplementary Table S1) across our panel of 25 clones. Not surprisingly, we observed a high degree of cross-correlation in expression of the measured genes (Figure 2b) likely due to the high interconnectivity in the circadian clock molecular loop. We subjected the dataset to Principal Component Analysis (PCA) aiming to extract the major features/genes that might underlie (or is a major contributor to) clonal-period heterogeneity. Based on the Broken-Stick model (Jolliffe , 2011), we retained the first two Principal Components (PCs) which collectively explained $70.2 \%$ of the circadian period variance (Figure $2 c$ ). Interestingly, the first two PCs also clustered the panel of clones into three categories of short (22.3-23h), intermediate (23.8-26.9h) and long (27.6-28.2h) period clones (Figure $2 \mathrm{~d}$ ). PC1 clustered the clones into two groups: i) intermediate periods and ii) the rest including both short and long period clones (nonintermediate). In contrast, PC2 appeared to be important for the three observed clusters (Figure $2 d$ ). Based on the $\cos ^{2}$ values (a measure of the quality of representation of the genes on a PC; Figure 2e-f) and contributions of genes to PC2 (Supplementary Figure S4), we shortlisted the top $25 \%$ of the candidate genes (ARNTL2, BHLHE40, DBP, NR1D2, PER2) that we hypothesized to largely account for the period-variation. 
138 We implemented hierarchical clustering on our dataset based on expression of the five shortlisted candidate genes and observed that clustering of clones was similar (with one exception) to that by the first two PCs (Figure $2 \mathrm{~g}$ ). The amalgamation schedule suggested a possibility of three clusters (red, blue and green dashed-rectangles, Figure $2 \mathrm{~g}$ ) which was also in agreement with the optimal cluster number reported by five different indices (Supplementary Figure S5). Clustering-based heat map revealed that the expression of ARNTL2 and BHLHE4O correlated positively with the circadian period, DBP and NR1D2 correlated negatively, while PER2 exhibited a clear trend (Figure 2g, Supplementary Figure S6a). As a control measure, we also similarly

147 shortlisted top 25\% genes from PC1 (NR1D1, CLOCK, CSNK1D, CIPC and NFIL3) and, as expected, 148 we observed that these genes were not sufficient to discriminate the short and long periods 149 thereby resulting in only two clusters - intermediate and non-intermediate (Figure $2 \mathrm{~h}$ ). Interestingly, all five genes from PC1 have higher expression in 'intermediate' period clones and 151 their expression reduces as the period deviates from 'intermediate' (Figure 2h, Supplementary 152 Figure S6b). Thus, we reasoned that changes in expression of PC2 genes are likely to drive period 153 heterogeneity while those from PC1 are likely to be a consequence of period heterogeneity.

154 We hypothesized that if differences in expression of the shortlisted PC2 genes governs period 155 heterogeneity, then depletion of these genes should result in large period change while depletion 156 of those from PC1 should not have a significant effect on period. Specifically, based on their expression patterns (Figure $2 \mathrm{~g}$ ) knockdown of $A R N T L 2$ and BHLHE4O should shorten the circadian period while $D B P$ and NR1D2 knockdown should result in period lengthening. To test this, we used RNAi mediated silencing to individually knockdown the shortlisted genes in 3-short, 2- 
intermediate and 3-long period clones (based on clustering in Figure $2 \mathrm{~g}$ ) and studied the effect on circadian period. Indeed, we observed that knockdown of NR1D2 resulted in significant period lengthening across all clones while BHLHE4O and ARNTL2 knockdown resulted in significant period shortening (Mixed model ANOVA followed by Tukey's HSD; $p<0.00001$; Figure 3a-b). NR1D2 knockdown had the largest effect on period, significantly higher compared to all other genes across both the PCs; followed by BHLHE4O that was similar to ARNTL2 and had a significantly higher effect on period compared to all other genes. Knockdown of none of the other genes across both PCs resulted in a period change significantly differing either from zero (one sample $t$ test, $p>0.05$ ) or from each other (Mixed model ANOVA followed by Tukey's HSD; $p>$ 0.05; Figure 3a-b). Accordingly, we observed that the average absolute period change upon knockdown of PC2 genes was significantly higher than that by PC1 genes (Figure 3c).

Taken together, these results suggest that differential expression of NR1D2, BHLHE4O and ARNTL2 likely underlies heterogeneity in clonal circadian period.

\section{Epigenetic regulation might underlie altered gene expression patterns associated with clonal} period-heterogeneity

Having observed that clonal period-heterogeneity is associated with altered gene expression patterns, we next asked 'what causes such altered expression among clonal cells?' We ruled out the possibility of random mutation accumulation (see Discussion) and hypothesized that epigenetic variation might account for the observed differences in gene expression patterns among clonal lines. As a preliminary test, we treated all 25 clonal cell populations in our panel with the commonly used epigenetic modifier Suberoylanilide Hydroxamic Acid (SAHA) and studied the effect of the treatment on clone period. SAHA is a Class I and Class II Histone 
182 Deacetylase Inhibitor which upregulates gene expression by multiple mechanisms (Marks et al.,

183 2008). We reasoned that if reduction in expression of the identified subset of genes across our

184 clonal panel is due to epigenetic suppression (in this case, acetylation status), treatment with

185 SAHA should upregulate the expression of these genes thereby lengthening and shortening the

186 circadian period in short and/or long period clones respectively.

187 Interestingly, we observed that treatment with SAHA differentially influenced the short, 188 intermediate and long period clones. SAHA treatment resulted in a significant period shortening 189 in the long period clones (ANOVA followed by Tukey's HSD, $p<0.05$; Figure $3 \mathrm{~d}$ ) whereas, the 190 magnitude of period change in short and intermediate period clones did not differ from each 191 other (ANOVA followed by Tukey's HSD, $p=0.85$ ) or from zero (one sample t test, $p>0.05$; Figure $1923 d$ ).

193 Although the possible reasons for the differential effects of SAHA treatment on short and long 194 period clones will be discussed later, this provides preliminary evidence suggesting that 195 epigenetically regulated gene expression differences might underlie clonal period-heterogeneity.

\section{DISCUSSION}

197 We used human U-2 OS cells to investigate whether period-heterogeneity in circadian clock 198 network stems from intrinsic/extrinsic non-heritable stochasticity or whether it has a heritable 199 component. We employed a laboratory selection protocol to select for clonal cell populations 200 exhibiting short and long circadian periods through which we derived a panel of 25 clonal cell 201 populations exhibiting circadian periods between $22 \mathrm{~h}$ to $28 \mathrm{~h}$. 
202 We observed that parental clones always produced progeny with mean periods closely

203

204

205

206

207

208

209

210

211

212

213

214

215

216

217

218

219

220

221

222

223

resembling the former thus resulting in a directional response (divergence of short and long period clones from the founding culture) to our selection protocol (Figure 1a). Consistently, the period of parental culture was a very good predictor $\left(R^{2}=0.96\right)$ of the progeny's mean period (Figure 1c). Taken together, these results suggest that clonal period-heterogeneity is unlikely to be stochastically driven and has a strong heritable component. This raises an interesting question: why would natural selection favour the evolution of heritable mechanisms to drive period-heterogeneity over entirely stochastically driven heterogeneity? We hypothesize that, although period heterogeneity can be functionally beneficial (Jagota et al., 2000; Schaap et al., 2003; Gonze et al., 2005; Bernard et al., 2007; Inagaki et al., 2007; VanderLeest et al., 2007; Gu et al., 2016, 2019), very large heterogeneity can negatively influence clock functionality as well (Gonze et al., 2005; Bernard et al., 2007; Gu et al., 2016). Stochastic mechanisms can potentially lead to very large variation in inter-cellular/oscillator period which would be detrimental, whereas heritable mechanisms may impose phenotypic constraints (Wagner, 2011) within which period-heterogeneity can be maintained and thus being favoured by natural selection.

Over the course of our experiments, we observed that long-period clones often exhibited higher bioluminescence intensity compared to the short-period clones (Supplementary Figure S2a) and further analysis revealed that parent bioluminescence intensity was a good predictor of progeny bioluminescence intensity but this was not the case for either relative amplitude or the damping rate (Figure 2a, Supplementary Figure S3). We reasoned that bioluminescence intensity could serve as a proxy measure for BMAL1 expression and thus hypothesised that period heritability is likely to be due to the inheritance of gene expression levels from parental clones. To further 
224 explore this, we measured average expression of 20 circadian clock and clock-associated genes

225 (Supplementary Table S1) across all 25 clones in our panel. By employing principal component 226 analysis, we identified five candidate genes (ARNTL2, BHLHE4O, DBP, NR1D2 and PER2) that

227 grouped the clones into three distinct clusters - short, intermediate and long periods (Figure 2c-

228 g, Supplementary Figures S4-5). Furthermore, we observed that knockdown of three of the

229 shortlisted candidates - NR1D2, BHLHE40 and ARNTL2 had the largest influence on period across

230 while other genes including those from PC1 had little or no effect on period change (Figure 3a-

231 c). It is noticeable that individual knockdown of the genes resulted in small magnitude period

232 changes that cannot entirely account or period differences between the short and long period

233 clones (Figure 3a). These results suggest that that clonal period-heritability is a multi-gene trait

234 involving a consortium of multiple medium-effect genes. Notably, all three above-mentioned

235 genes are transcription factors that are either regulated by and/or act on E-boxes and are part of

236 both the core and auxiliary molecular clock loops (Ikeda et al., 2000; Okamura et al., 2002;

237 Kawamoto et al., 2004; Guillaumond et al., 2005; Nakashima et al., 2008; Sasaki et al., 2009;

238 Takahashi, 2017). This reinforces the idea that while persistence of circadian oscillation requires

239 a functional core clock loop involving negative feedback by the PER-CRY family, modulation of

240 clock period might be governed by interaction between multiple loops coupled by E-box

241 associated transcription factors (Zhang and Kay, 2010; Relógio et al., 2011). Another notable gene

242 that our analysis revealed happens to be one of the relatively less studied circadian clock genes

243 ARNTL2 (BMAL2). While ARNTL2 is a functional paralog of the core clock gene ARNTL1 (BMAL1),

244 its precise role in the clock loop remains largely elusive (Ikeda et al., 2000; Sasaki et al., 2009; Shi 
245 et al., 2010) thus highlighting a potential role of ARNTL2 in circadian period-modulation, which

246 awaits further exploration.

247 Intriguingly, in contrast to the above-discussed genes, we find another category among the 248 assayed genes that exhibit an inverted-U shaped relationship with period. The expression of 249 these genes (NR1D1, CSNK1D, NFIL3, CLOCK, CIPC) is high in clones with intermediate periods $250(23.8-26.9 \mathrm{~h})$ and is drastically reduced in clones with periods deviating from the intermediate 251 range (Figure 3g). Furthermore, our knockdown studies also confirm that expression patterns of 252 these genes are not causal but likely to be a response/consequence to period variation (Figure 3b). Such inverted-U shaped responses (Hormesis) is observed in various biological systems and

254 is regarded as a regulatory/homeostatic mechanism to prevent very large deviations of 255 cellular/organismal phenotypes from their optimal range (Calabrese and Baldwin, 2001; Baldi 256 and Bucherelli, 2005; Zhang et al., 2008). As discussed earlier, since a higher degree of period257 heterogeneity can be detrimental to the circadian clock network, we hypothesize that while there 258 are mechanisms within the clock circuitry that promote period-heterogeneity, the network might 259 also harbour hormesis-based mechanisms which impose constraints on the range of period that 260 the circadian clock can exhibit (Baldi and Bucherelli, 2005; Zhang et al., 2008). Such mechanisms 261 may also explain why we observe a saturation of period divergence after assay generation 2 262 (Figure 1b).

263 While evidence thus far strongly suggests that clonal period-heterogeneity is driven by 264 differences in clock gene expressions, we then asked 'what is the source of these expression 265 differences?' One possibility is that the short and long period clones might have accumulated 266 random mutations resulting in period change and subsequently selected by us. However, we 
267 reason that this is highly unlikely because - a) With a mutation rate of $\sim 2.5 \times 10^{-8} /$ nucleotide in

268 human cells (Nachman and Crowell, 2000), the probability of occurrence of at least two kinds of

269 mutations within a small fraction of the genome (comprising clock genes) driving short and long

270 periods is extremely low. b) We see significant trends in expression of the same subset of genes

271 across both short and long period clones (Figure 2g; Supplementary Figure S6). This presupposes

272 that mutations driving short and long periods have occurred within the same genes, which

273 further drastically reduces the probability that the observed period differences stem from

274 random mutations. c) Even if the mutation rate is higher than we estimate, the saturation of

275 divergence in period over the last three assay generations (Figure 1b) cannot be entirely

276 accounted by mutations since the periods could continue to diverge due to further accumulation

277 of mutations. Therefore, we argue that the observed period-heterogeneity is unlikely to be due

278 to random mutations, which leaves us with another alternative - epimutations. Epimutations are

279 heritable changes in expression of genes and are not associated with DNA mutations.

280 Epimutations are often associated with changes in methylation states of genes or other heritable

281 chromatin modifications (Holliday, 2006). The rates of epimutations are observed to be order of

282 magnitude higher than DNA mutation rates (Van Der Graaf et al., 2015) and successfully explains

283 phenotypic heterogeneity in many life forms including clonal populations (Kaufmann et al., 2007;

284 Stockholm et al., 2007; Neildez-Nguyen et al., 2008; Taudt et al., 2016; Springer and Schmitz,

285 2017). Therefore, we hypothesized that epimutations-driven gene expression differences may

286 underlie clonal heterogeneity in circadian period. As a preliminary test of this hypothesis, we

287 studied the effect of a Histone Deacetylase Inhibitor Suberoylanilide Hydroxamic Acid (SAHA)

288 treatment on the circadian period across our clones. Interestingly, we find that treatment with 
SAHA significantly shortens (albeit by a small magnitude) the period in long-period clones with little or no effect on the short and intermediate clones (Figure 3d). The small magnitude effect of SAHA treatment might be due to one or all of the following reasons. SAHA is broad spectrum Histone Deacetylase (HDAC) inhibitor and promotes upregulation of genes by acetylation, whereas other epigenetic mechanisms that might contribute to the gene expression in our clones are not targeted by this treatment. Alternatively, off-target effects of SAHA might also upregulate

295 other genes that in turn negatively influence the change in period. In addition, as discussed previously, if period heterogeneity is indeed a multi-gene trait relying on combined upregulation and downregulation of two or more genes, mere treatment with epigenetic modifiers that leads to genome-wide changes in gene expression may not be a good strategy. Nevertheless, the differential effects of SAHA on short and long period clones is promising and provides preliminary support to the idea that epigenetic modulation of gene expression might underlie clonal periodheterogeneity. Future targeted studies along these lines may shed more light on this aspect. networks in mammals is not stochastically driven but has a heritable basis and that this is likely to be a multi-gene trait. We identified that differential regulation of three E-box associated transcription factors might govern period-heterogeneity in circadian clock networks and provide preliminary evidence that epigenetically regulated gene expression differences may underlie clonal period-heterogeneity. In addition, we also observed a subset of genes that exhibit which we hypothesize are part of homeostatic mechanisms that may constrain circadian clocks from deviating largely from their optimal period range. Future studies will help further explore the phenomenon of period-heterogeneity and its regulation. 


\section{MATERIALS AND METHODS}

\section{Clone selection protocol}

313 All clones used in this study were U-2 OS cells (human, ATCC \# HTB-96) stably expressing firefly

314 luciferase from a 0.9-kb BMAL1 promoter (Maier et al., 2009), cultured and maintained in DMEM

315 containing $10 \%$ fetal bovine serum, antibiotics (100 U/ml penicillin and $100 \mu \mathrm{g} / \mathrm{ml}$ streptomycin).

316 See Supplementary Figure S1 for a pictorial description of the selection protocol. Briefly, cells

317 from 'founding culture' expressing a circadian period of $24.6 \pm 0.16 \mathrm{~h}$ (mean \pm SD) were plated as

318 single-cell clones in 96-well 'parent plates' and grown to confluency. Upon reaching confluency,

319 an 'assay plate' was established for every 'parent plate' by splitting cells. The period of

320 bioluminescence rhythms from cells in 'assay plates' was recorded (see below for recording

321 protocol) and clones exhibiting short or long periods (tails of the period-distribution) were

322 selected. Bioluminescence rhythms of every clone was recorded 2-3 times and only clones that

323 consistently exhibited shot/long periods were selected. Following the selection of clones,

324 corresponding clones from the 'parent plate' were single-cell cloned in 96-well plates, and the

325 procedure was repeated for four assay generations by selecting short and long period clones

326 every generation.

\section{Bioluminescence recording}

328 Cells were plated in white 96 -well plate at a density of $20 \times 10^{3}$ cells/well and after 72 hours, cells

329 were synchronized with dexamethasone $(1 \mu \mathrm{M})$ for 30 minutes, washed twice with PBS and

330 cultured in Phenol-Red-free DMEM containing 10\% fetal bovine serum, antibiotics (100 U/ml

331 penicillin and $100 \mu \mathrm{g} / \mathrm{ml}$ streptomycin) and $250 \mu \mathrm{M}$ D-luciferin (Biothema, Darmstadt, Germany). 
Bioluminescence was recorded at $37^{\circ} \mathrm{C}$ in a 96 -well plate luminescence counter (TopCount,

used for data analysis and estimation of rhythms parameters including period, decay constant (damping), relative amplitude and average bioluminescence (MESOR) of the oscillation as described previously (Abraham et al., 2010).

\section{RNA preparation and NanoString based gene expression analysis}

338 Five days before the RNA extraction, cells were plated at a density of $\sim 20 \times 10^{3}$ cells/well in 24 well plate with DMEM containing $10 \%$ fetal bovine serum, antibiotics $(100 \mathrm{U} / \mathrm{ml}$ penicillin and $100 \mu \mathrm{g} / \mathrm{ml}$ streptomycin). Since we intended to measure average gene expression levels, the culture medium was not replaced for five days to prevent accidental synchronization of cells. On

342 day-5 the medium was removed, $100 \mu \mathrm{l} /$ well iScript ${ }^{\mathrm{TM}}$ RT-qPCR Sample Preparation Reagent

343 (Biorad) was added on top of the cell-layer and incubated at $37^{\circ} \mathrm{C}$ for $5 \mathrm{~min}$. $3 \mu \mathrm{l}$ of the sample was withdrawn without disturbing the cell-layer and used for further downstream analysis as per manufacturer's instructions.

A previous study of ours combined whole-genome transcriptomics with machine learning and identified genes that could serve as reliable circadian time-telling markers (Wittenbrink et al., 2018). Based on this, we designed a 24-plex NanoString probe panel comprising 20 circadian clock 349 and clock associated genes and 4 housekeeping genes (Supplementary Table S1). The custom350 designed probes included a 3 '-end biotinylated capture probe and a 5 '-fluorescence-barcoded 351 reporter probe for each gene target. Hybridization of probes and gene expression-count reading 352 was according to the manufacturer's instructions. Raw expression data was acquired by a 
353 NanoString nCounter Digital Analyzer (NanoString Technologies), QC processed and analysed by

354 nSolver ${ }^{T M}$. QC analysis flagged reads from one (CIART) of the 24 genes in the panel as unsuitable

355 for analysis and was not considered. Data normalization involved three steps: (a) normalization

356 by the arithmetic mean of the positive spike-in controls, (b) subtraction of the mean of the

357 negative controls, and (c) normalization by the geometric mean of the four housekeeping genes.

\section{Principal Component Analysis and Clustering}

$359 \log _{2}$-transformed gene expression data were first subjected to Bartlett's Test of Sphericity to 360 validate its adequacy for Principal Component Analysis (PCA) following which correlation-based 361 PCA was implemented in R (R Core Development Team, 2013) using factoextra and FactoMineR 362 packages (Kassambara, 2016). Broken-Stick model (Jolliffe, 2011) was used to determine the 363 number of retainable Principal Components (PCs). Determining the optimal cluster-number is 364 often a complication in unsupervised exploratory data analysis. Unlike many studies in biology 365 that employ PCA to identify genes based on expression differences between known cell-types

366 (which can be used to estimate the optimal number of clusters), our study employs a panel of 367 clones with a continuous distribution of phenotypes (period) and thus cannot be categorized 368 trivially. Hence, we adopted two schemes for optimal cluster-number determination. (a) For 369 agglomerative hierarchical clustering, we assessed the agglomeration schedule to identify the 370 possible number of clusters (Yim and Ramdeen, 2015). (b) In addition, we also performed k371 means clustering for different values of cluster $(k=1-10)$ and used 5 different indexes 372 'silhouette method' (Rousseeuw, 1987), 'elbow method' (Thorndike, 1953), 'gap-statistic' 373 (Tibshirani et al., 2001), 'Calinski-Harabasz criterion value (variance-ratio method)' (Caliñski and 374 Harabasz, 1974) and Bayesian Information Criterion (BIC; Fraley and Raftery, 2002) to assess the 
375 optimal cluster-number. We selected the optimal cluster number based on agreement between

376 (a) and (b). Heatmapper (Babicki et al., 2016) and 'dendextend' (Galili, 2015) were used for

377 hierarchical clustering analysis based on 'euclidean-distance' and 'complete-linkage' measures

378 (D, 2005). 'Nbclust' (Charrad et al., 2014) and 'mclust' (Scrucca et al., 2016) were used for k-

379 means based clustering analysis while for all other statistical analysis and graphing was

380 performed using R and Prism version 8.00 for Windows (GraphPad Software, La Jolla California

381 USA, www.graphpad.com).

\section{RNAi mediated gene knockdown}

383 The GIPZ microRNA-adapted shRNA constructs used for the study were purchased from Open

384 Biosystems and packaged into lentiviral vectors in HEK293T cells in a 96-well plate format (Maier

385 et al., 2009). Virus-containing supernatants were then filtered and reporter cells (clonal cell

386 populations used in the study) were transduced with $150 \mu \mathrm{L}$ of the filtrate containing $8 \mathrm{ng} / \mu \mathrm{L}$

387 protamine sulfate. After at least $24 \mathrm{~h}$, the filtrate was replaced with fresh medium containing

388 puromycin $(10 \mu \mathrm{g} / \mathrm{mL})$. After 3 days, the transduced reporter cells were synchronized and

389 bioluminescence rhythms were recorded as described above.

\section{SAHA treatment and dose response analysis}

$39110^{3}$ cells were plated in a 96 -well plate on day-0. After $24 \mathrm{~h}$, the culture media was replaced with

392 media containing 1.6 $\mu \mathrm{M}$ Suberanilo Hydroxamic Acid (SAHA) or DMSO vehicle control. The drug

393 was replaced every day for three consecutive days. On day-4, the cells were rinsed thrice with

394 PBS and fresh (no drug) culture media was added. The cells were untreated for next $48 \mathrm{~h}$ and

395 bioluminescence rhythms were recorded from day-6. 
396 The above-described protocol was followed for estimating the $\mathrm{IC}_{50}$ value as well. Cells were

397 treated with varying concentrations $(0-100 \mu \mathrm{M})$ of SAHA from day-1, and cell proliferation was

398 assayed on day- 6 using the Vybrant ${ }^{\circledR}$ MTT Cell Proliferation Assay Kit (Thermo Fischer Scientific,

399 catalog \#V13154) as per manufacturer's protocol. IC 50 was calculated from the resulting dose

400 response curve using Prism version 8.00 for Windows (GraphPad Software, La Jolla California

401 USA, www.graphpad.com; Supplementary Figure S7).

402

403 ACKNOWLEDGEMENTS

404 The authors thank Sebastian Jäschke and Katja Schellenberg, Astrid Grudziecki and Almut Eisele

405 for their help with single-cell cloning; Hedwig Lammert, Michael Hummel and Barbara Koller for

406 help with NanoString experiments and Lucas Hille for his help with gene-knockdown and

407 epigenetics related experiments. Work in AK's lab is supported by grants from the Deutsche

408 Forschungsgemeinschaft to SK (SFB740/D2) and AK (TRR186/P17). Alexander von Humboldt-

409 Stiftung supported NKL's research.

410

411 AUTHOR CONTRIBUTIONS

412 Conceptualization: NKL, SK and AK; Experiments and data acquisition: NKL and SK. Data curation

413 and formal analysis: NKL and SK; Manuscript preparation: NKL and AK.

414

415 COMPETING INTERESTS

416 The authors declare no conflict of interest. 


\section{REFERENCES}

419 Abraham, U., Granada, A.E., Westermark, P.O., Heine, M., Kramer, A. and Herzel, H., 2010.

420 Coupling governs entrainment range of circadian clocks. Molecular systems biology, 6(1).

421 Altschuler, S.J. and Wu, L.F., 2010. Cellular heterogeneity: do differences make a difference?.

422 Cell, 141(4), pp.559-563.

423 Babicki, S., Arndt, D., Marcu, A., Liang, Y., Grant, J.R., Maciejewski, A. and Wishart, D.S., 2016.

424 Heatmapper: web-enabled heat mapping for all. Nucleic acids research, 44(W1), pp.W147-

425 W153.

426 Baldi, E. and Bucherelli, C., 2005. The inverted "u-shaped" dose-effect relationships in learning

427 and memory: modulation of arousal and consolidation. Nonlinearity in biology, toxicology,

428 medicine, 3(1), pp.nonlin-003.

429 Bell-Pedersen, D., Cassone, V.M., Earnest, D.J., Golden, S.S., Hardin, P.E., Thomas, T.L. and

430 Zoran, M.J., 2005. Circadian rhythms from multiple oscillators: lessons from diverse

431 organisms. Nature Reviews Genetics, 6(7), p.544.

432 Bernard, S., Gonze, D., Čajavec, B., Herzel, H. and Kramer, A., 2007. Synchronization-induced

433 rhythmicity of circadian oscillators in the suprachiasmatic nucleus. PLoS computational

434 biology, 3(4), p.e68.

435 Brock, A., Chang, H. and Huang, S. (2009) 'Non-genetic heterogeneity a mutation-independent 436 driving force for the somatic evolution of tumours', Nature Reviews Genetics, 10(5), pp. 336$437 \quad 342$. 
438

439

440

441

442

443

444

445

446

447

448

449

450

451

452

453

454

455

Calabrese, E. J. and Baldwin, L. A. (2001) 'U-Shaped Dose-Responses in Biology, Toxicology, and Public Health', Annual Review of Public Health, 22(1), pp. 15-33.

Caliñski, T. and Harabasz, J. (1974) 'A Dendrite Method Foe Cluster Analysis', Communications in Statistics, 3(1), pp. 1-27.

Charrad, M. et al. (2015) 'NbClust : An R Package for Determining the Relevant Number of Clusters in a Data Set ', Journal of Statistical Software, 61(6).

Dubnau, D. and Losick, R., 2006. Bistability in bacteria. Molecular microbiology, 61(3), pp.564572.

Dunlap, J. C. (1999) 'Molecular Bases for Circadian Clocks', Cell, 96(2), pp. 271-290.

Evans, C. R. et al. (2018) ‘Errors during gene expression: Single-cell heterogeneity, stress resistance, and microbe-host interactions', mBio. American Society for Microbiology, 9(4), pp. e01018-18. doi: 10.1128/mBio.01018-18.

Fowlkes, E.B. and Mallows, C.L., 1983. A method for comparing two hierarchical clusterings. Journal of the American statistical association, 78(383), pp.553-569.

Fraley, C. and Raftery, A. E. (2002) 'Model-based clustering, discriminant analysis, and density estimation', Journal of the American Statistical Association, 97(458), pp. 611-631.

Frank, S.A. and Rosner, M.R., 2012. Nonheritable cellular variability accelerates the evolutionary processes of cancer. PLoS biology, 10(4), p.e1001296.

Galili, T. (2015) 'dendextend: An R package for visualizing, adjusting and comparing trees of hierarchical clustering', Bioinformatics, 31(22), pp. 3718-3720. 
458

459

460

461

462

463

464

465

466

467

468

469

470

471 Van Der Graaf, A. et al. (2015) 'Rate, spectrum, and evolutionary dynamics of spontaneous

472 epimutations', Proceedings of the National Academy of Sciences of the United States of

473 America, 112(21), pp. 6676-6681. doi: 10.1073/pnas.1424254112.

474 Gu, C., Liang, X., Yang, H. and Rohling, J.H., 2016. Heterogeneity induces rhythms of weakly

475 coupled circadian neurons. Scientific reports, 6, p.21412. 
476 Gu, C., Wang, P., Weng, T., Yang, H. and Rohling, J., 2019. Heterogeneity of neuronal properties

477 determines the collective behavior of the neurons in the suprachiasmatic

478 nucleus. Mathematical biosciences and engineering: MBE, 16(4), pp.1893-1913.

479 Guillaumond, F., Dardente, H., Giguère, V. and Cermakian, N., 2005. Differential control of

480 Bmal1 circadian transcription by REV-ERB and ROR nuclear receptors. Journal of biological

481 rhythms, 20(5), pp.391-403.

482 Holliday, R., 2006. Epigenetics: a historical overview. Epigenetics, 1(2), pp.76-80.

483 Huang, S., 2009. Non-genetic heterogeneity of cells in development: more than just

484 noise. Development, 136(23), pp.3853-3862.

485 Ikeda, M., Yu, W., Hirai, M., Ebisawa, T., Honma, S., Yoshimura, K., Honma, K.I. and Nomura, M.,

486 2000. cDNA cloning of a novel bHLH-PAS transcription factor superfamily gene, BMAL2: its

487 mRNA expression, subcellular distribution, and chromosomal localization. Biochemical and

488 biophysical research communications, 275(2), pp.493-502.

489 Inagaki, N., Honma, S., Ono, D., Tanahashi, Y. and Honma, K.I., 2007. Separate oscillating cell

490 groups in mouse suprachiasmatic nucleus couple photoperiodically to the onset and end of

491 daily activity. Proceedings of the National Academy of Sciences, 104(18), pp.7664-7669.

492 Jagota, A., De La Iglesia, H. O. and Schwartz, W. J. (2000) 'Morning and evening circadian

493 oscillations in the suprachiasmatic nucleus in vitro', Nature Neuroscience, 3(4), pp. 372-376.

494 Jolliffe, I., 2011. Principal component analysis (pp. 1094-1096). Springer Berlin Heidelberg. 
495

496

497

498

499

500

501

502

503

504

505

506

507

508

509

510

511

512

513

Kassambara, A. (2017) Practical Guide To Principal Component Methods in R: PCA, M (CA), FAMD, MFA, HCPC, factoextra. STHDA.

Kaufmann, B.B., Yang, Q., Mettetal, J.T. and van Oudenaarden, A., 2007. Heritable stochastic switching revealed by single-cell genealogy. PLoS biology, 5(9), p.e239.

Kawamoto, T., Noshiro, M., Sato, F., Maemura, K., Takeda, N., Nagai, R., Iwata, T., Fujimoto, K., Furukawa, M., Miyazaki, K. and Honma, S., 2004. A novel autofeedback loop of Dec1

transcription involved in circadian rhythm regulation. Biochemical and biophysical research communications, 313(1), pp.117-124.

Kumar, V. (ed.) (2017) Biological timekeeping: Clocks, rhythms and behaviour, Biological Timekeeping: Clocks, Rhythms and Behaviour. Springer Nature.

Leise, T.L., Wang, C.W., Gitis, P.J. and Welsh, D.K., 2012. Persistent cell-autonomous circadian oscillations in fibroblasts revealed by six-week single-cell imaging of PER2:: LUC bioluminescence. PloS one, 7(3), p.e33334.

Lopez-Molina, L., Conquet, F., Dubois-Dauphin, M. and Schibler, U., 1997. The DBP gene is expressed according to a circadian rhythm in the suprachiasmatic nucleus and influences circadian behavior. The EMBO Journal, 16(22), pp.6762-6771.

Maier, B., Wendt, S., Vanselow, J.T., Wallach, T., Reischl, S., Oehmke, S., Schlosser, A. and Kramer, A., 2009. A large-scale functional RNAi screen reveals a role for CK2 in the mammalian circadian clock. Genes \& development, 23(6), pp.708-718. 
514 Marks, P.A., Richon, V.M., Miller, T. and Kelly, W.K., 2004. Histone deacetylase

515 inhibitors. Advances in cancer research, 91, pp.137-168.

516 Muranaka, T. and Oyama, T., 2016. Heterogeneity of cellular circadian clocks in intact plants

517 and its correction under light-dark cycles. Science advances, 2(7), p.e1600500.

518 Nachman, M.W. and Crowell, S.L., 2000. Estimate of the mutation rate per nucleotide in

519 humans. Genetics, 156(1), pp.297-304.

520 Nagoshi, E., Saini, C., Bauer, C., Laroche, T., Naef, F. and Schibler, U., 2004. Circadian gene

521 expression in individual fibroblasts: cell-autonomous and self-sustained oscillators pass time to

522 daughter cells. Cell, 119(5), pp.693-705.

523 Nakashima, A., Kawamoto, T., Honda, K.K., Ueshima, T., Noshiro, M., Iwata, T., Fujimoto, K.,

524 Kubo, H., Honma, S., Yorioka, N. and Kohno, N., 2008. DEC1 modulates the circadian phase of

525 clock gene expression. Molecular and cellular biology, 28(12), pp.4080-4092.

526 Neildez-Nguyen, T.M.A., Parisot, A., Vignal, C., Rameau, P., Stockholm, D., Picot, J., Allo, V., Le

527 Bec, C., Laplace, C. and Paldi, A., 2008. Epigenetic gene expression noise and phenotypic

528 diversification of clonal cell populations. Differentiation, 76(1), pp.33-40.

529 Okamura, H., Yamaguchi, S. and Yagita, K., 2002. Molecular machinery of the circadian clock in

530 mammals. Cell and tissue research, 309(1), pp.47-56.

531 Patrik, D. (2005) 'How does gene expression clustering work ?’, Nature Biotechnology, 23(12),

532 pp. 1499-1501. 
533 R Core Team (2018) 'R: A language and environment for statistical computing'. Vienna: R

534 Foundation for Statistical Computing. Available at: https://www.r-project.org/.

535 Relógio, A., Westermark, P.O., Wallach, T., Schellenberg, K., Kramer, A. and Herzel, H., 2011.

536 Tuning the mammalian circadian clock: robust synergy of two loops. PLoS computational

537 biology, 7(12), p.e1002309.

538 Roberfroid, S., Vanderleyden, J. and Steenackers, H., 2016. Gene expression variability in clonal

539 populations: causes and consequences. Critical reviews in microbiology, 42(6), pp.969-984.

540 Rousseeuw, P.J., 1987. Silhouettes: a graphical aid to the interpretation and validation of cluster

541 analysis. Journal of computational and applied mathematics, 20, pp.53-65.

542 Sabado, V., Vienne, L. and Nagoshi, E. (2017) 'Evaluating the Autonomy of the Drosophila

543 Circadian Clock in Dissociated Neuronal Culture', Frontiers in Cellular Neuroscience, 11(317), pp.

544 1-10.

545 Sasaki, M., Yoshitane, H., Du, N.H., Okano, T. and Fukada, Y., 2009. Preferential inhibition of

546 BMAL2-CLOCK activity by PER2 reemphasizes its negative role and a positive role of BMAL2 in

547 the circadian transcription. Journal of Biological Chemistry, 284(37), pp.25149-25159.

548 Schaap, J., Albus, H., Tjebbe vanderLeest, H., Eilers, P.H., Détári, L. and Meijer, J.H., 2003.

549 Heterogeneity of rhythmic suprachiasmatic nucleus neurons: Implications for circadian

550 waveform and photoperiodic encoding. Proceedings of the National Academy of

551 Sciences, 100(26), pp.15994-15999. 
552 Scrucca, L., Fop, M., Murphy, T.B. and Raftery, A.E., 2016. mclust 5: clustering, classification and 553 density estimation using Gaussian finite mixture models. The R journal, 8(1), p.289.

554 Shi, S., Hida, A., McGuinness, O.P., Wasserman, D.H., Yamazaki, S. and Johnson, C.H., 2010.

555 Circadian clock gene Bmal1 is not essential; functional replacement with its paralog,

556 Bmal2. Current biology, 20(4), pp.316-321.

557 Springer, N. M. and Schmitz, R. J. (2017) ‘Exploiting induced and natural epigenetic variation for 558 crop improvement', Nature Reviews Genetics, 18(9), pp. 563-575.

559 Stockholm, D., Benchaouir, R., Picot, J., Rameau, P., Neildez, T.M.A., Landini, G., Laplace-Builhé, 560 C. and Paldi, A., 2007. The origin of phenotypic heterogeneity in a clonal cell population in 561 vitro. PloS one, 2(4), p.e394.

562 Takahashi, J. S. (2017) 'Transcriptional architecture of the mammalian circadian clock', Nature 563 Reviews Genetics, 18(3), pp. 164-179.

564 Snedecor, W. G. and Cochran, G. W. (1989) Statistical Methods. 8th edn. Iowa State University 565 Press.

566 Taudt, A., Colomé-Tatché, M. and Johannes, F. (2016) 'Genetic sources of population

567 epigenomic variation', Nature Reviews Genetics, 17(6), pp. 319-332.

568 Thorndike, R. L. (1953) 'Who belongs in the family?', Psychometrika, 18(4), pp. 267-276.

569 Tibshirani, R., Walther, G. and Hastie, T. (2001) 'Estimating the number of clusters in a data set 570 via the gap statistic', Journal of Royal Statistical Society B, 63(2), pp. 411-423. 
571 Van Der Graaf, A., Wardenaar, R., Neumann, D.A., Taudt, A., Shaw, R.G., Jansen, R.C., Schmitz,

572 R.J., Colomé-Tatché, M. and Johannes, F., 2015. Rate, spectrum, and evolutionary dynamics of

573 spontaneous epimutations. Proceedings of the National Academy of Sciences, 112(21), pp.6676-

5746681.

575 VanderLeest, H.T., Houben, T., Michel, S., Deboer, T., Albus, H., Vansteensel, M.J., Block, G.D.

576 and Meijer, J.H., 2007. Seasonal encoding by the circadian pacemaker of the SCN. Current

577 Biology, 17(5), pp.468-473.

578 Wagner, A., 2011. Genotype networks shed light on evolutionary constraints. Trends in Ecology

579 \& Evolution, 26(11), pp.577-584.

580 Welsh, D.K., Logothetis, D.E., Meister, M. and Reppert, S.M., 1995. Individual neurons

581 dissociated from rat suprachiasmatic nucleus express independently phased circadian firing

582 rhythms. Neuron, 14(4), pp.697-706.

583 Wittenbrink, N., Ananthasubramaniam, B., Münch, M., Koller, B., Maier, B., Weschke, C., Bes, F.,

584 de Zeeuw, J., Nowozin, C., Wahnschaffe, A. and Wisniewski, S., 2018. High-accuracy

585 determination of internal circadian time from a single blood sample. The Journal of clinical

586 investigation, 128(9), pp.3826-3839.

587 Yakir, E., Hassidim, M., Melamed-Book, N., Hilman, D., Kron, I. and Green, R.M., 2011. Cell

588 autonomous and cell-type specific circadian rhythms in Arabidopsis. The Plant Journal, 68(3),

589 pp.520-531. 
590 Yim, O. and Ramdeen, K.T., 2015. Hierarchical cluster analysis: comparison of three linkage

591 measures and application to psychological data. The quantitative methods for

592 psychology, 11(1), pp.8-21.

593 Zhang, E.E. and Kay, S.A., 2010. Clocks not winding down: unravelling circadian

594 networks. Nature reviews Molecular cell biology, 11(11), p.764.

595 Zhang, Q., Pi, J., Woods, C.G., Jarabek, A.M., Clewell III, H.J. and Andersen, M.E., 2008. Hormesis

596 and adaptive cellular control systems. Dose-Response, 6(2), pp.dose-response. 


\section{FIGURE LEGENDS}

601 Figure 1: Clonal period-heterogeneity is not stochastically driven but largely inherited

602 (a) Divergence of the period-distributions of short (red) and long (blue) period clones from a

603 common founding culture (green) across multiple assay generations. Dashed black lines depict

604 the mean of respective period distributions. The grey dashed line extended from assay

605 generation-1 depicts mean period of the founding culture (assay generation 0 ) for visual

606 assessment of the period divergence. Red (short period clone) and blue (long period clone)

607 arrows indicate the means periods of representative clones selected for the successive

608 generation (b) Divergence of the mean period among three representative clonal lines each for

609 long-period clonal line (LCL) 1-3 and short-period clonal line (SCL) 1-3. Error bars are SD ( $n=3-5$

610 experiments). $\not x$ indicates that the period of all three SCLs differs significantly from all three LCLS

611 for the given assay generation. Asterisks $(*)$ on top represent LCLs and those at the bottom

612 represent SCLs. Asterisks of different colours indicate that the period of the three clones in that

613 generation is significantly different $(p<0.001 ; n=3-5)$ from the periods in other generations,

614 while those with the same colour do not differ significantly. For example, the periods of SCLs 1-3

615 in assay generation-2 differ significantly from their periods in assay generation-1 and from the

616 founding culture in assay generation-0, but not from assay generations 3 and 4. (c) Regression of

617 progeny cultures' periods on mean periods of their parental cultures' periods as a proxy-estimate

618 of heritability. Each data point is an average of 3-5 experiments. Blue solid line is the linear

619 regression fit with its $95 \% \mathrm{Cl}$ (blue dotted line).

620 
621 Figure 2: Inheritance of clock-gene expression patterns might govern clonal period622 heterogeneity

623 (a) Linear regression of progeny mean bioluminescence intensity on parental values suggests a

624 strong heritability of mean bioluminescence intensity $\left(R^{2}=0.76\right)$. Each data point is an average 625 of 3-5 experiments. Blue solid line is the linear regression fit with its $95 \% \mathrm{Cl}$ (blue dotted line). (b)

626 Cross-correlation of average expression values between the 19 analysed genes across all 25

627 clones indicates a high degree of inter-gene correlation. The colour and size of the circles

628 represent the strength of correlation (Pearson $r$ ). (c) Scree plot depicting the percentage of

629 variance explained by the 19 principal components (black bars) and the expected values based

630 on the Broken-Stick model (red line). (d) Factor map of individual clones plotted across the

631 principal components (PCs) 1 and 2 reveals that the first two PCs clusters the clones in three 632 clusters of short (red), intermediate (green) and long (blue) period clones. (e) Correlation circle 633 depicting the loading of 19 genes across PCs 1 and 2. (f) $\operatorname{Cos}^{2}$ values (squared loadings as a 634 measure of the quality of representation of a gene on a PC) of the 19 genes for PCs 1-5. The 635 colour and size of circles represent the magnitude of $\operatorname{Cos}^{2}$ value. (g) Hierarchical clustering based 636 on the expression of 5 genes selected from PC2. With the exception of one clone, all others 637 clustered into 3 groups of short, intermediate and long clones (red, green and blue dashed 638 rectangles respectively). (h) Hierarchical clustering based on the expression of 5 genes from PC1 639 resulted in 2 clusters - i) intermediate period (green dashed rectangle) and ii) short and long 640 period (blue dashed rectangle). The colour coding of clones in $(\mathrm{g})$ and $(\mathrm{h})$ is the same as in (d). 
642 Figure 3: Epigenetically regulated expression of E-Box associated factors may govern clonal 643 period-heterogeneity

644 Period change (compared to non-silenced control) upon knockdown of the (a) five PC2 genes and

645 (b) three PC1 genes for the short (red), intermediate (green) and long (blue) period clones. Bars

646 with different symbols indicate significant differences $(p<0.05)$ while bars with same symbols

647 are not significantly different from each other (Mixed model ANOVA followed by Tukey's HSD).

648 (c) Averaged absolute period change across all clones upon knockdown of genes from PC2 (black)

649 and PC1 (grey). (d) Period change (compared to vehicle control) upon treatment of short (red),

650 intermediate (green) and long (blue) period clones with HDAC (Histone Deacetylase) inhibitor

651 SAHA (1.6 $\mu \mathrm{M})$. For all panels in this figure, $\mathrm{n}=3-4$ experiments and error bars are SD. *: $p<0.05$;

$652 * * * *: p<0.0001$

653 


\section{Figure 1}

(a)

${ }^{50}$ Founding Culture ; Assay Generation 0

bioRxiv preprint doi: https://doi.org/10. $\$ 1 / 73407$; this version posted September 11, 2019. The copyright holder for this preprint (which was not certified by peer Eyiew) is the author/funder. All (ights reserved. No reuse allowed without permission.
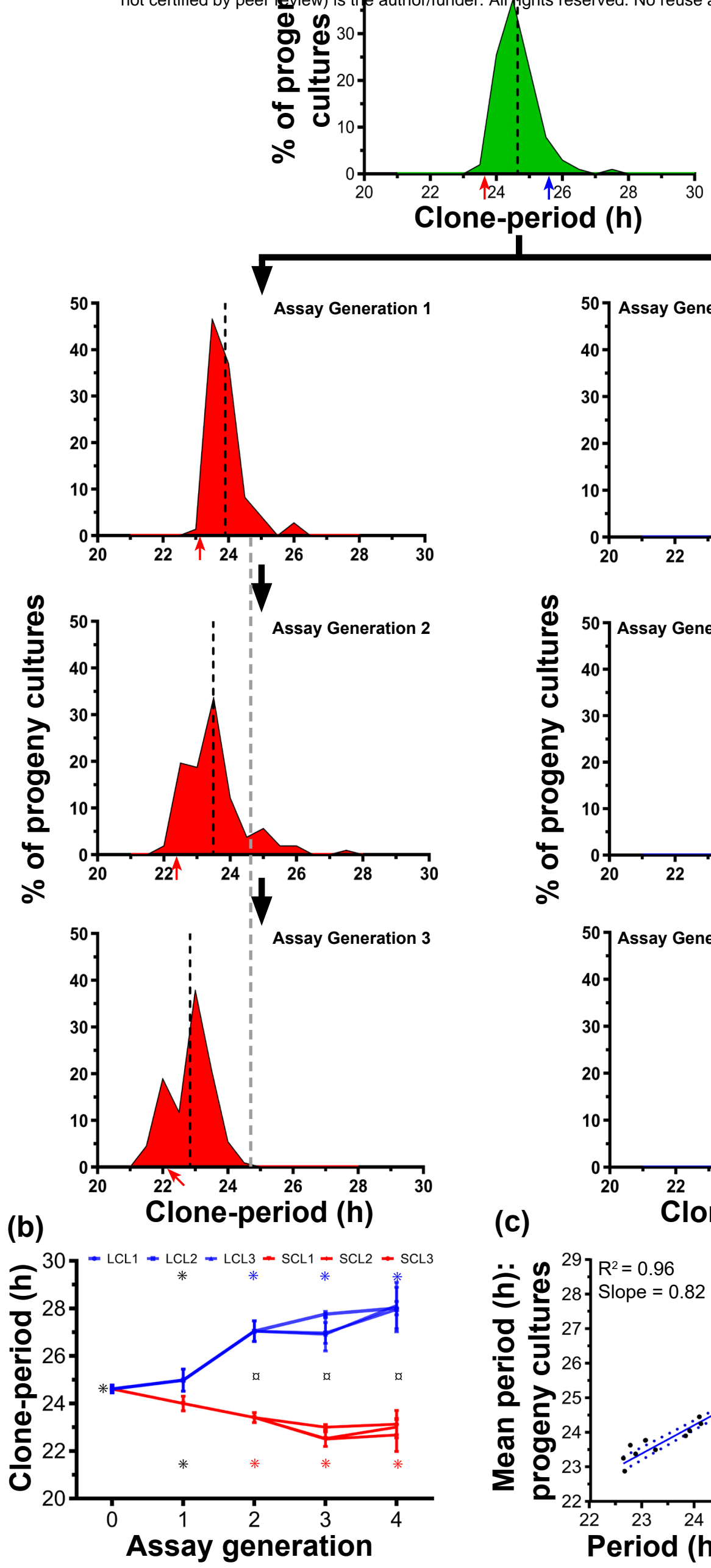

(c)
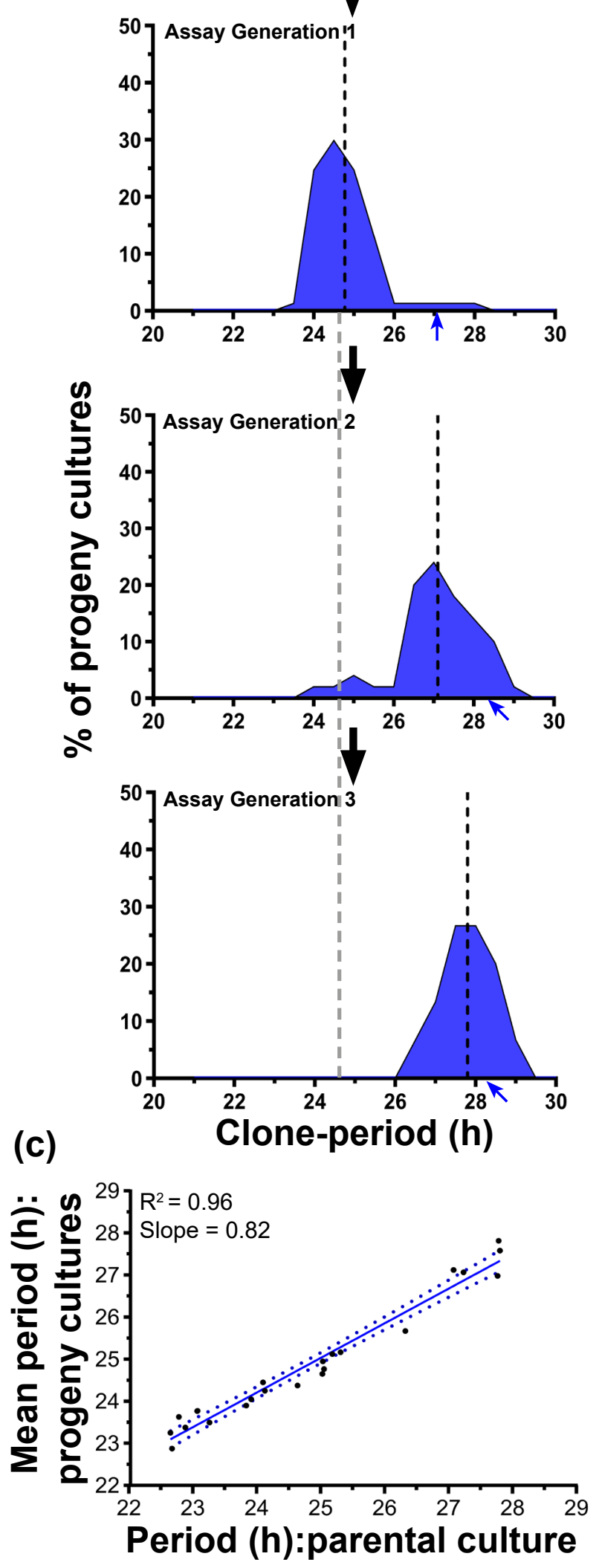


\section{Figure 2}

bioRxiv preprint doi: https://doi.org/10.1101/731075; this version posted September 11, 2019. The copyright holder for this preprint (which was not certified by peer review) is the author/funder. All rights reserved. No reuse allowed without permission.

(a)

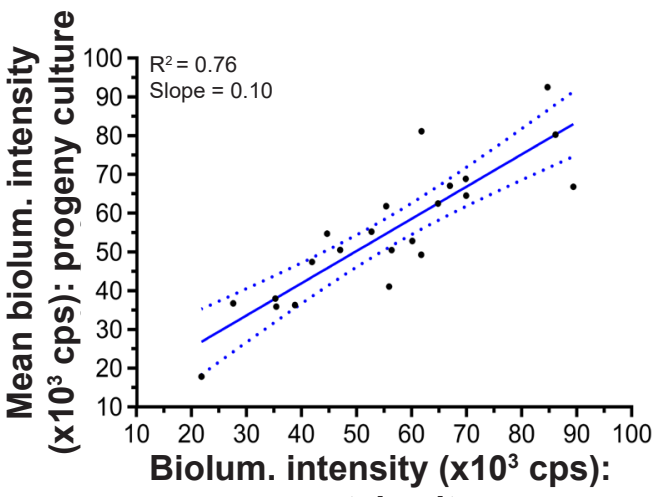

parental culture

(d)

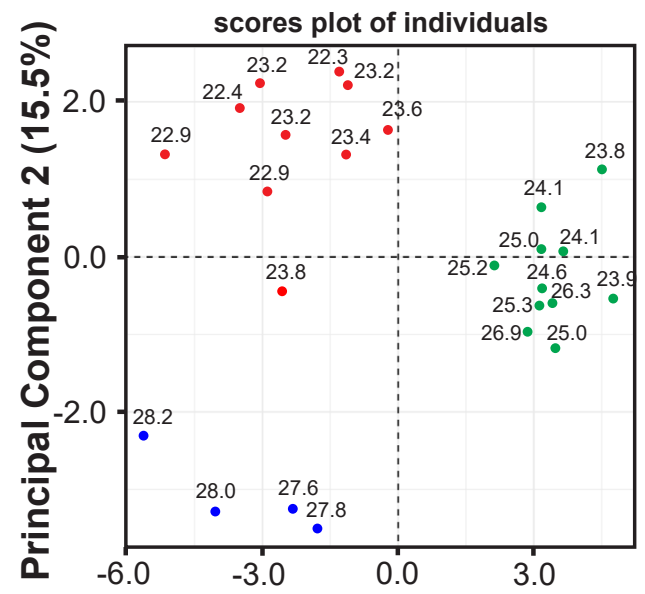

(b)

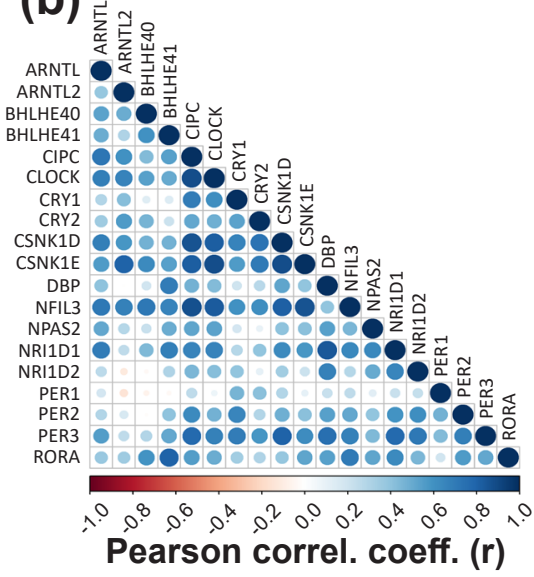

(e)

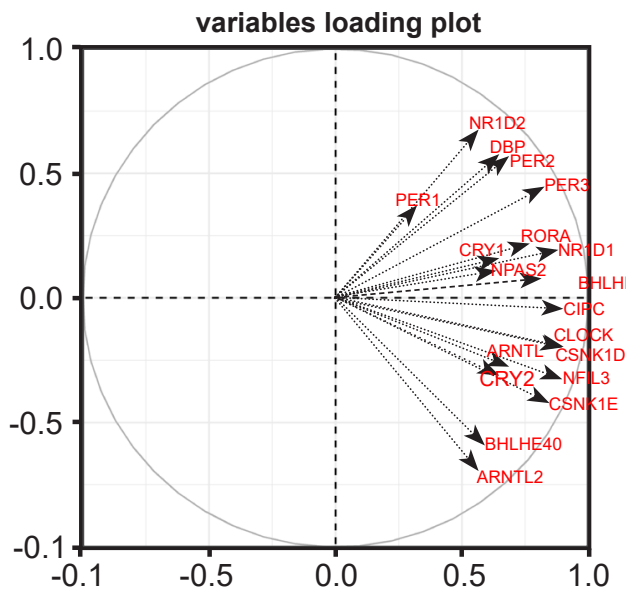

(c)

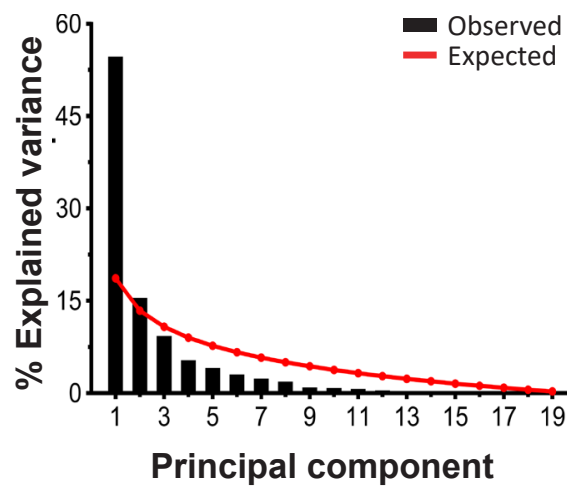

(f)

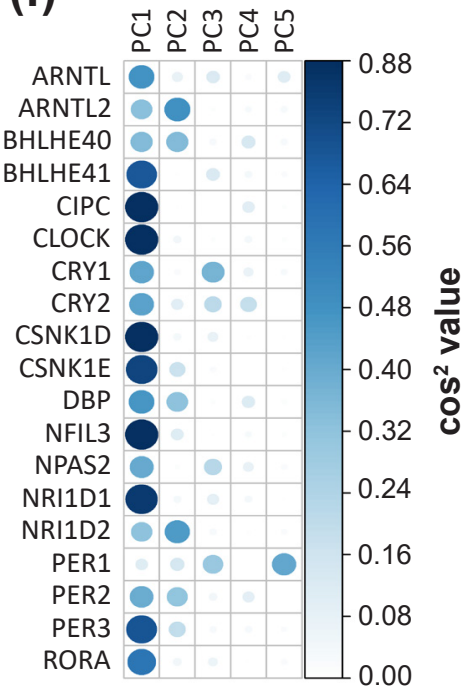

(g)

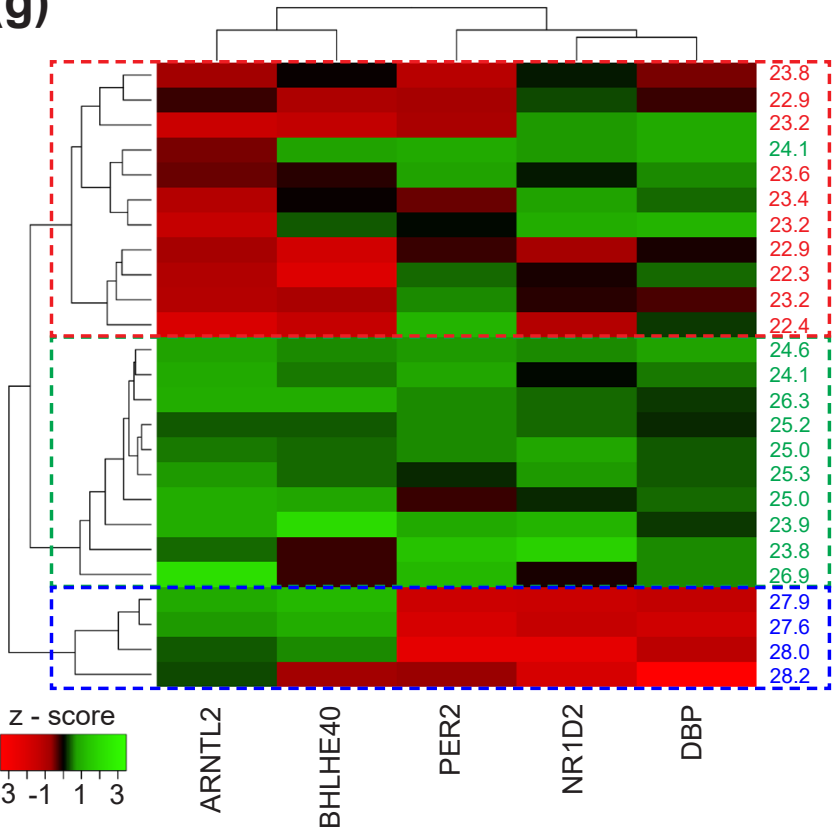

(h)

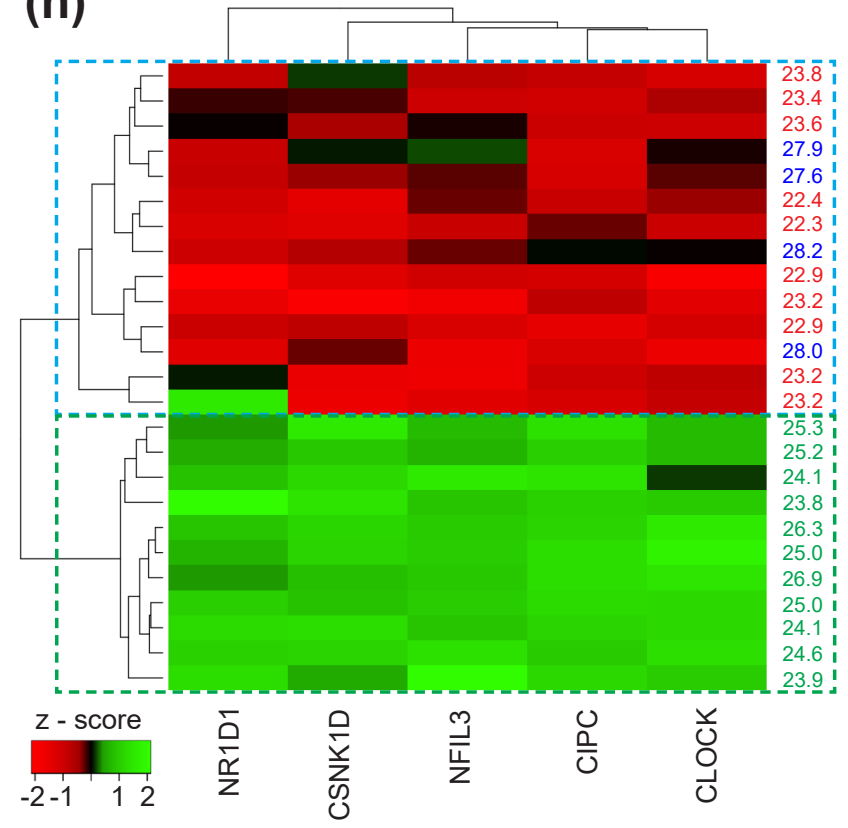


Figure 3

bioRxiv preprint doi: https://doi.org/10.1101/731075; this version posted September 11, 2019. The copyright holder for this preprint (which was not certified by peer review) is the author/funder. All rights reserved. No reuse allowed without permission.

(a)

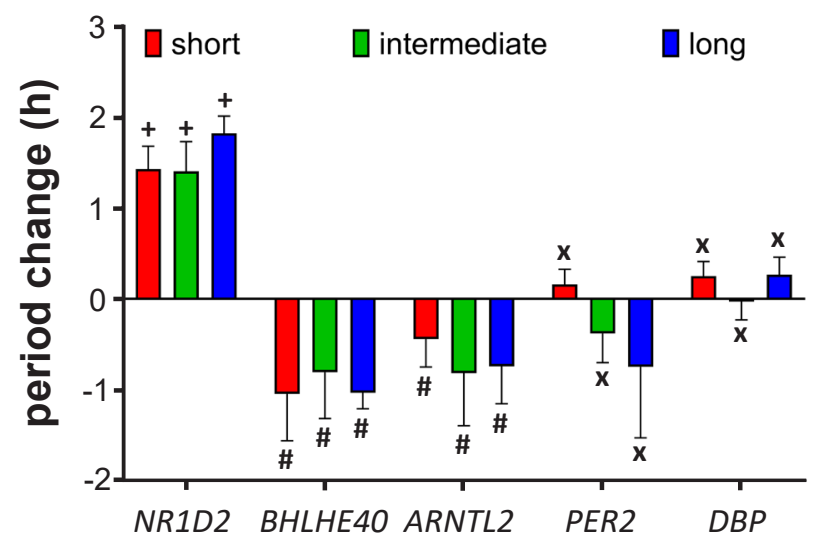

(c)

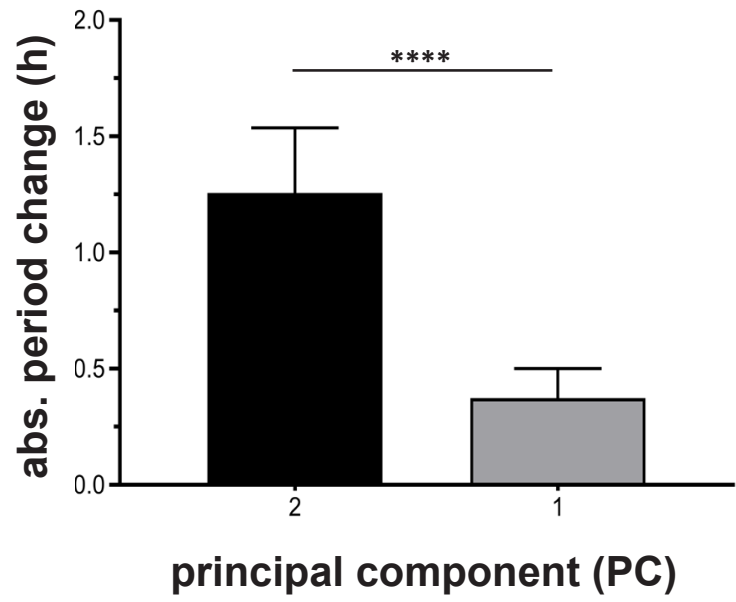

(b)

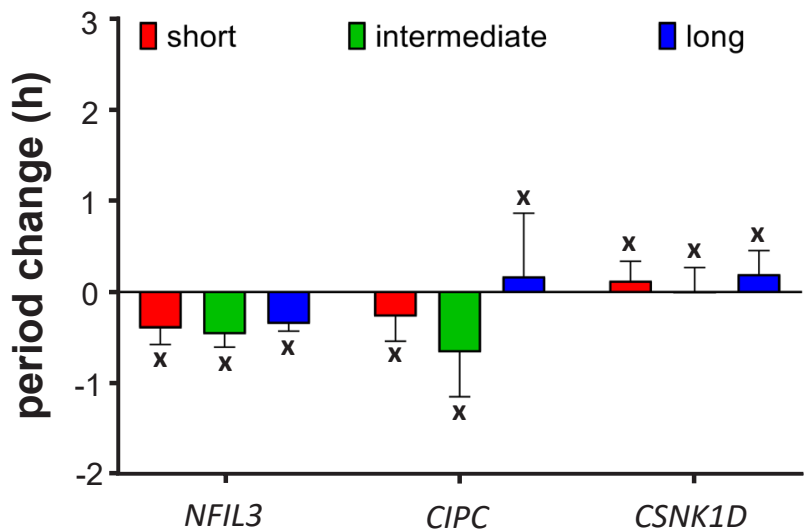

(d)

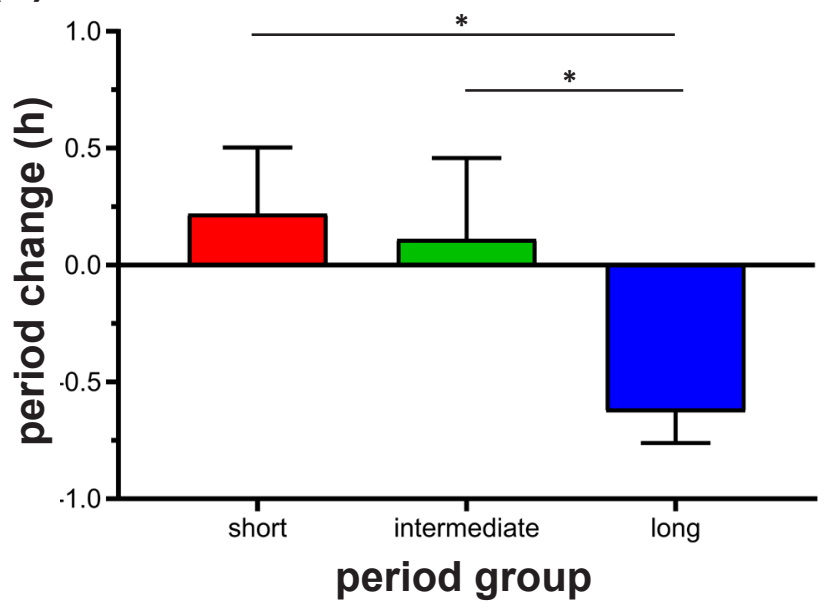

